\title{
UNA RARA AVIS EN LOS ALBORES DE LA DEMOCRACIA: ESPECIFICIDADES Y CARACTERÍSTICAS DE LA IZQUIERDA EN FUERTEVENTURA (1977-1982)
}

\author{
Ángel Dámaso Luis León* \\ Universidad de La Laguna
}

\section{ResUmen}

La izquierda majorera de los inicios de la democracia se organizó de una manera muy particular. Con escasa implantación de los partidos de la izquierda tradicional y con el surgimiento de nuevas formas políticas a la sombra del cambio de régimen, se produjeron una serie de resultados y tendencias bastante llamativos. Entre ellos, el más llamativo fue el surgimiento y la cuota de poder alcanzado por parte de Asamblea Majorera, primer partido insularista con representación política en Espańa. En este artículo, se analizará las divergencias que presenta la izquierda majorera en los inicios del período democrático y los diferentes proyectos que surgen a raíz de esas divergencias.

Palabras Clave: historia electoral, izquierda, Canarias, insularismo, Fuerteventura, Asamblea Majorera, PSOE.

\section{A RARA AVIS AT THE BEGINNING OF DEMOCRACY: SPECIFICITIES AND}

CHARACTERISTICS OF THE LEFTIST PARTIES IN FUERTEVENTURA (1977-1982)

\section{Abstract}

The left of the beginnings of Democracy was organized in a very particular way on Fuerteventura. With little implementation of the parties of the traditional left and with the emergence of new political forms in the shadow of the change of regime, a series of results and quite striking tendencies took place. Among them, the most remarkable was the emergence and the power reached by Asamblea Majorera, the first insularist party with political representation in Spain. In this paper, we will analyze the divergences that this left presents at the beginning of Democracy and the different projects that arise and how they develop. KeYwords: electoral history, left-wing, Canary Islands, insularism, Fuerteventura, Asamblea Majorera, PSOE. 


\section{INTRODUCCIÓN}

¿Fue Fuerteventura una realidad diferente durante los inicios de la democracia en Canarias y España? Esa es una de las preguntas principales que se busca resolver en este artículo. La aparición de una izquierda asamblearia e insularista potente, unida al escaso peso de los partidos tradicionales de este ámbito ideológico nos lleva a plantear la hipótesis de que así fue, pero la clave es ise sostiene esta afirmación ante un proceso de análisis y estudio crítico en profundidad?

A través de la lectura y análisis de los datos electorales que se produjeron entre los años 1977 y 1982, en los diferentes procesos electorales acaecidos, se intentará presentar y analizar cómo se canalizó el apoyo izquierdista en la isla. Para posicionar bien los fenómenos que se producen, no solo se deberán desglosar y analizar los resultados, sino también compararlos y contrastarlos con otros contextos asimilables al majorero. Por ello, la utilización de una metodología comparativa mostrará si realmente Fuerteventura fue una rara avis durante los primeros años de la democracia o si existe algún otro fenómeno similar en algún contexto comparable.

Para ello, además del prolífico y relativamente sencillo acceso a la información electoral, se deberá acometer una lectura de otro tipo de fuentes históricas que apoyan el proceso de investigación. Entre ellas destacan las fuentes de tipo hemerográfico, que nos aportan datos e impresiones sobre acontecimientos acaecidos en la época. Dicha información posee numerosas limitaciones de sobra conocidas, pero, aun así, la seguimos considerando la principal fuente para la historia de este proceso, ${ }^{1}$ debido a la escasez de fuentes de archivo, por el fallecimiento de informantes que permitan hacer un estudio de historia oral escasamente sesgado y relevante $y$, principalmente, por su propio valor intrínseco como fuente histórica ${ }^{2}$, la cual se encuentra fuera de toda duda ${ }^{3}$. El mayor problema de este tipo de fuentes para el caso de la Transición en Fuerteventura, más allá de los ya típicamente conocidos para la prensa como fuente en general, es el tradicional centralismo de la estructura mediática en Canarias durante los primeros años del experimento democrático. Años en los que la gran mayoría de las publicaciones periódicas se situaban en las dos islas capitalinas (con alguna tímida excepción), dejando para la información de las llamadas «islas menores» o periféricas un espacio menor y menos relevante, cuanti-

* Doctorando en el Programa de Doctorado Islas Atlánticas: Historia, Patrimonio y Marco Jurídico-Institucional. Contratado predoctoral del Área de Historia de América de la Universidad de La Laguna. Fundación Cajacanarias-Caixabank. Correo electrónico: aluisleo@ull.edu.es.

${ }^{1}$ Hernández Ramos, Pablo: «Consideración teórica sobre la prensa como fuente historiográfica», Historia y Comunicación Social, n. ${ }^{\circ} 22$, vol. 2, 2017, pp. 465-477.

${ }^{2}$ Llegados a este punto resulta imprescindible reconocer la contribución de dos herramientas insalvables que ponen a disposición del investigador un inmenso tesoro hemerográfico. Por una parte la hemeroteca de la Universidad de La Laguna y su herramienta en línea Prensa Canaria Digitalizada y, por otra Jable. Archivo de Prensa Digital de la Biblioteca Universitaria de la Universidad de Las Palmas de Gran Canaria. Esta investigación no ha sido una excepción y debe agradecer mucho a ambas instituciones.

${ }^{3}$ Hernández Ramos, Pablo: op. cit. 
tativa y cualitativamente. En ese sentido, resulta necesario resaltar algunos trabajos realizados por Mario Ferrer Peñate, quien ha estudiado tanto la prensa local en esos años ${ }^{4}$ como las corresponsalías de prensa en las islas menores orientales (Fuerteventura y Lanzarote) durante este período5.

Esta obra también cuenta con otra limitación con la que contrastar datos, hipótesis e información, y es la escasa literatura científica publicada hasta la época. Esta sufre dos limitaciones principales. Por un lado, hay una escasa bibliografía sobre aspectos concretos, y la existente se restringe a cuestiones específicas como el estudio de las corresponsalías mencionado en el párrafo anterior, o a cuestiones relacionadas con algún partido en concreto ${ }^{6}$. El otro principal problema a abordar son las escasas alusiones a la realidad majorera que suelen presentar los estudios más genéricos y que aspiran a dar una visión de todo el archipiélago, sobre todo en cuestiones de tipo político, y que suelen caer en idéntica tentación centralista que las publicaciones periódicas anteriormente mencionadas.

Una vez presentadas las fuentes, se necesita establecer un marco que acote la investigación. Aunque no sea este el lugar adecuado para una diatriba teórica sobre el desarrollo del concepto Transición, es necesario mostrar las cartas, temporales y contextuales, sobre las que se parte. Aunque hay otros autores que dan cronologías más estrechas o más amplias para la Transición española, en este trabajo aceptamos la secuencialización que realiza Javier Tusell para este período ${ }^{7}$, y que sitúa el principio y el fin del proceso entre los años 1975 y 1982, entre la muerte del dictador Francisco Franco y el cambio acaecido en el Gobierno español, que pasó de las manos de la UCD a las del PSOE.

Llegados a este punto y una vez definidos los límites del trabajo, resulta lícito, honesto y necesario exponer una limitación infranqueable que esta investi-

${ }^{4}$ Ferrer Peñate, Mario: El periodismo en la periferia de Europa Occidental. Prensa, sociedad y opinión pública en Lanzarote y Fuerteventura (1852-1982), San Cristóbal de La Laguna, tesis doctoral inédita de la Universidad de La Laguna, 2012, pp. 260-287.

5 Ferrer Peñate, Mario: «Corresponsalías de prensa y política durante la Transición en Lanzarote y Fuerteventura», en León Álvarez, Aarón (coord.): La Transición en Canarias. Actas del Encuentro de Historia sobre la Transición en Canarias: del tardofranquismo a la democracia, 19691986, La Orotava. San Cristóbal de La Laguna, Le Canarien Ediciones. Instituto de Estudios Canarios, pp. 221-241.

${ }^{6}$ Solamente Asamblea Majorera, como experiencia atípica, se puede salvar de esta tónica general, contando con dos publicaciones principales. La primera, más temprana y de un tipo más cronístico, fue publicada por Bermúdez Suárez y Torres Torres en el año 2003 (y editada por la propia AM). Y la segunda, más puramente científica, fue presentada en las Jornadas de Estudios sobre Fuerteventura y Lanzarote, celebradas en 2015 y cuya publicación se ha retrasado finalmente hasta 2020. Las referencias bibliográficas son Bermúdez Suárez, Felipe; Torres Torres, Carmelo C.: Asamblea Majorera: 25 años de historia, Puerto del Rosario, Asamblea Majorera, 2003; Luis León, Ángel Dámaso: «Asamblea Majorera: Bagaje e historia electoral de un insularismo de izquierdas", en XVI Jornadas de Estudios sobre Fuerteventura y Lanzarote, Puerto del Rosario, Cabildo de Fuerteventura, 2020 [en prensa].

7 Tusell, Javier: Dictadura franquista y Democracia, 1939-2004, Barcelona, Editorial Crítica, 2010, pp. 277-331 
gación posee, y que no es otra que la incapacidad para establecer mejores descripciones sociológicas de la población majorera en ese entonces. Esta es una limitación potente, ya que solo la podemos estimar a través del voto. La dolorosa (pero lógica) ausencia de estudios sociológicos sobre la población limita a la hora de construir una imagen más fehaciente de la realidad de la época.

En definitiva, este trabajo lo que busca es plantear, con todas sus limitaciones, si la izquierda majorera presentaba diferenciación o no dentro de su ámbito contextual, y, si es así, en qué grado lo hacía y cómo se expresaba políticamente.

\section{EL MONOPOLIO DE ASAMBLEA MAJORERA}

Asamblea Majorera surge como un partido asambleario en los albores de la democracia. Se podría decir que es el primer partido netamente insular e insularista exitoso en Espańa, puesto que obtiene grandes éxitos electorales desde los primeros comicios que se celebran en el país, ya en el año 1977. Ideológicamente es una agrupación realmente compleja de definir: nace con una vocación puramente asamblearia pero pronto se erigen algunos liderazgos importantes que en ocasiones rayan lo personalista. Dentro del eje izquierda-derecha posee una vocación de izquierdas, con tintes nacionalistas, y con variaciones temporales, pero sobre todo se proyecta de manera insularista ${ }^{8}$.

Algunos especialistas en la cuestión, como Hernández Bravo de Laguna, no identifican a AM como un fenómeno insularista, sino como un fenómeno político influenciado por el mismo9. En este trabajo no aceptamos esta definición porque no se considera al insularismo periférico una mera expresión posterior y elitista del llamado pleito insular (el pleito entre Gran Canaria y Tenerife), sino que este insularismo se expresa y articula de manera autónoma. Tampoco el insularismo, en su conformación necesaria, debe plantearse como una expresión elitista, al menos no en su desarrollo, puede tener otro tipo de conformaciones. Para afirmar esto, nos fundamentamos en la definición de insularismo heredada del párrafo anterior y, sobre todo, de la tradición puramente histórica de este tipo de expresiones.

La tradición de vindicación insular (porque afirmar que «insularista», para esos tiempos, podría no ajustarse a la realidad histórica) de Fuerteventura tenía un notable componente previo. El llamado «Plebiscito de las islas menores» del año 1910 y la posterior Ley de Cabildos de dos años más tarde (1912), habían recibido una profunda influencia de posicionamientos que en la isla majorera tenían cierto

${ }^{8}$ El insularismo puede ser definido como una readaptación del regionalismo a un entorno fragmentado. Este asume la existencia de una comunidad política superior, no desde el plano de la alteridad, como en el nacionalismo, sino en el de la pragmática expresión de un mejor gobierno generado por la cercanía. Para ampliar esta definición puede verse Luis León, Ángel Dámaso: op. cit., 2020, pp. 3-4.

9 Hernández Bravo de Laguna, Juan: «El insularismo canario: caracterización política, ofertas electorales y resultados», Papers. Revista de Sociología, n. ${ }^{\circ} 33,1990$, pp. 123-124. 
calado. Sin ir más lejos, uno de los roles más importantes durante todo este proceso de auge del pensamiento y posterior concreción de una anhelada autonomía insular lo había jugado el majorero Miguel Velázquez Cabrera, considerado por la historiografía tradicional como uno de los «padres» de la estructura institucional cabildicia surgida de la segunda década del siglo xx (junto al palmero Pedro Pérez Díaz) $^{10}$, y que, en cierta medida, aunque con profundas modificaciones en su vocación y esencia, sobrevive hasta hoy.

Partiendo de esta base histórica, el estudio de AM resultaría profundamente necesario, puesto que sería la constatación principal de dos ideas. La primera es que existe y es efectivamente la articulación de un insularismo de izquierdas con una potencia electoral destacable. Y la segunda es que sería la primera expresión partidista exitosa de ese tipo. Antecediendo al momento clave del boom del insularismo canario en la etapa democrática, que se produce tras la implosión de la Unión de Centro Democrático en el archipiélago ${ }^{11}$, y a la conformación de otros grupos también previos a esa implosión como AHI.

Con esa tradición previa al franquismo no resulta descabellado que existiera un magma del que nace AM. Y lo hace como una continuación del llamado Grupo Democrático Majorero, un grupo de personalidades e intelectuales que se reunían para discutir acerca de cuestiones políticas ya en los últimos años del período franquista y que deciden presentarse al Senado en los comicios de 1977. Este proyecto pronto recibe amplios apoyos por parte de la sociedad civil y políticos y consigue sobrevivir a algunas pequeñas escisiones, ganando finalmente dichos comicios al Senado, donde Miguel Cabrera ejercerá como el primer senador majorero de la democracia ${ }^{12}$. No presentarán candidatura al Congreso de los Diputados porque era electoralmente inviable ${ }^{13}$ es más, es el partido de ámbito más pequeño cuyo

${ }^{10}$ La historiografía canaria, sobre todo la de finales del siglo xx, retrató y reinterpretó de manera constante la problemática insular y su reflejo institucional a principios de siglo. El llamado "problema canario", los diferentes "pleitos» insulares y la articulación de la región han llenado multitud de páginas en publicaciones con forma de libros y artículos en revistas especializadas. Citarlos todos no es el ejercicio de este trabajo, que se centra en el último cuarto del siglo xx y no en el primero; sin embargo, una buena forma de abordarlos es a través de la publicación de Manuel SARmiento Acosta: «1912-2012: hechos, hombres e instituciones con ocasión de la Ley de Cabildos Insulares», Tebeto. Anuario del Archivo Histórico Insular de Fuerteventura, n. ${ }^{\circ}$ 21, 2016, pp. 187-228; quien realiza un análisis de gran parte de la producción existente hasta la actualidad.

11 Báez García, Alberto Javier: «Unión de Centro Democrático (UCD). El partido de la Transición en Canarias (1977-1982)», en León Álvarez, Aarón (coord.): La Transición en Canarias. Actas del Encuentro de Historia sobre la Transición en Canarias: del tardofranquismo a la democracia, 1969-1986, La Orotava. San Cristóbal de La Laguna: Le Canarien Ediciones. Instituto de Estudios Canarios, 2018, pp. 385-401.

12 Bermúdez Suárez, Felipe y Torres Torres, Carmelo C.: op. cit., pp. 20-27.

13 Aunque la isla de Fuerteventura tenga en la actualidad más población que alguna provincia española (más de 110000 habitantes) y un peso relativo en la provincia de Las Palmas por encima del $10 \%$, el crecimiento de esta población se ha producido de manera muy reciente. A finales de los setenta la población total no llegaba a los 30000 habitantes. Según los cálculos de Álvarez Alonso, Hernández Hernández y Simancas Cruz la isla tenía 27104 habitantes, mientras que Gon- 
candidato resulta electo en esos primeros comicios. La movilización social, canalizada de manera asamblearia en gran medida, sería importante, pero esta victoria, además de ello, tendría un notable efecto a posteriori sobre el partido, ya que resultaría clave para que el electorado se convenciera de que AM era un proyecto electoralmente viable.

El año 1979 sería el de la consagración definitiva del partido, a pesar de presentar unos resultados agridulces. Por una parte, Cabrera no podría revalidar su asiento en la Cámara alta tras perder las elecciones por una diferencia de solo una decena de votos con respecto al candidato de la UCD, Miguel Sánchez Velázquez ${ }^{14}$. Este hecho estuvo propiciado fundamentalmente por el hecho de que había dos candidaturas de izquierdas al Senado (la de AM y la del PSOE), mientras que en el año 1977, Cabrera había aglutinado todo el apoyo progresista en la isla.

En la Cámara baja, en cambio, se llevarían una alegría indirecta. Esta llegaría a través de la consecución por parte de la UPC, en la figura de Fernando Sagaseta, de un escaño en el Congreso de los Diputados por la provincia de Las Palmas, partido para el que AM pediría el voto en esta elección. Aunque, todo sea dicho, la contribución del voto en bruto majorero a esta empresa fue realmente pequeña.

Una de las principales curiosidades que propició la aparición con fuerza de AM es convertir a Fuerteventura en un lugar con un porcentaje elevadísimo de split ticket. El split ticket puede ser definido como la acción por parte del elector de votar a dos partidos políticos diferentes en votaciones que se producen de manera simultánea. Lo contrario sería el straight ticket ${ }^{15}$. Lo que presumiblemente no observamos en el caso majorero, al menos en los inicios de la democracia, es la aparición de un alto grado de "voto dual», es decir, votar a un partido nacional en elecciones de ámbito nacional y a un partido de ámbito no estatal (en adelante PANE o PANES en plural), como es el caso de AM, en comicios destinados a elegir representantes

zález Morales aporta unos datos algo mayores, situando la cifra total en 30185 habitantes. Ambos guarismos son para el ańo 1981, por lo que inferimos que durante los ańos anteriores, una isla en pleno crecimiento demográfico marcará indicadores más exiguos. Para ese mismo año de 1981, el peso relativo de la isla majorera apenas suponía el $4 \%$ del peso total provincial, lo que invalidaba una candidatura competitiva al Congreso. Datos extraídos de Álvarez Alonso, Antonio; Hernández Hernández, Jesús y Simancas Cruz, Moisés R.: «Transformaciones recientes en la distribución territorial de la población de Canarias», en Cuadernos Geográficos, n. ${ }^{\circ} 36$, vol. 1, 2005, p. 351; GonzÁlez Morales, Alejandro: «La población de la isla de Fuerteventura, 1857-2001», en Tebeto. Anuario del Archivo Histórico Insular de Fuerteventura, n. ${ }^{\circ}$ 17, 2004, p. 342; y del ISTAC, con cálculos propios.

${ }_{14}$ Resultados para el Senado en la isla de Fuerteventura (marzo de 1979): 1. ${ }^{\circ}$ Miguel Sánchez Velázquez (UCD) 4468 votos; $2 .^{\circ}$ Miguel Cabrera Cabrera (AM) 4458 votos; 3. José Francisco Jorge Machín (CD) 650 votos; 4. ${ }^{\circ}$ Manuel Hernández Hierro (PSOE) 341 votos. Datos del Ministerio del Interior.

15 Nohlen, Dieter: Sistemas electorales y partidos politicos. México D.F.: Fondo de Cultura Económica, 1996, p. 84. 
para una institución más pequeña ${ }^{16}$. La división, como se podrá observar a continuación, es más entre papeletas que entre elecciones.

Tras la dolorosa derrota en las elecciones al Senado de 1979, resulta llamativa la rapidez con la que se produce la recuperación del partido y cómo, lejos de hundirse por el varapalo y por la posible fragilidad de la experiencia, se recupera en los siguientes comicios, celebrados apenas un mes después.

La importancia de los mismos es que se dirimía el futuro de dos tipos de instituciones diferentes, ambas de carácter local y ambas elegidas democráticamente por primera vez. Entrar en ellas suponía implantarse definitivamente en la gestión insular. El 3 de abril de 1979, los majoreros estaban llamados a dotar de representantes a su Cabildo Insular y a los diferentes ayuntamientos de la isla. En el ámbito municipal, AM consigue varios resultados importantes, mayormente en el sur de la isla, principal granero histórico de votos de la agrupación. Logra vencer en los dos municipios más meridionales de la isla: Tuineje y Pájara, consiguiendo una amplia mayoría de concejales en ambos. Además de estas mayorías, el partido sería fuerza principal de la oposición en el resto de municipios, ya fuera con su nombre propio o con otros adaptados para la ocasión. El ámbito local marcaría una tendencia que se mantendría durante los siguientes años: el sur de la isla tendería a votar por opciones más de izquierdas, el norte lo haría por formaciones de derechas.

Carlos Cabrera Ajeno sería el primer alcalde electo de Pájara y Paco Hierro el de Tuineje, ${ }^{17}$ pero no serían las únicas cabezas visibles de AM en las instituciones majoreras. Si el resultado en los comicios locales fue bueno, más lo sería el de las elecciones a la corporación insular.

Gerardo Mesa Noda sería el primer candidato de AM al Cabildo. Empleado de banca y hombre cercano a los cristianos de base, no solo se convertiría en el primer candidato de la agrupación insularista, sino que además llegaría a ser el primer presidente del Cabildo majorero democráticamente electo. Lográndolo además con mayoría absoluta y sorteando las múltiples dificultades que aparecieron durante la campaña electora ${ }^{18}$. Las primeras elecciones municipales e insulares confirmaban lo que ya habían dejado entrever las generales de 1977, AM era un partido electoralmente muy fuerte, y había llegado a las instituciones para quedarse. El insularismo gozaba de una estupenda salud en Fuerteventura, siendo AM la principal representación política no solo de los electores que defendían este tipo de posicionamientos, sino también del electorado que se consideraba de izquierdas.

Como se puede observar en la figura n. ${ }^{\circ} 1^{19}$, solo hubo una isla donde los proyectos insulares pudieron igualar los resultados aparecidos en Fuerteventura: el

${ }^{16}$ Pallarés, Francesc, Montero Gibert, José Ramón y Llera Ramo, Francisco José: «Non State-Wide Parties in Spain: An Attitudinal Study of Nationalism and Regionalism», en Publius: the journal of federalism, $n$. $^{\circ} 27$, vol. 4, 1997, pp. 166-168.

17 Bermúdez Suárez, Felipe y Torres Torres, Carmelo C.: op. cit., p. 41.

${ }^{18}$ Luis León, Ángel Dámaso: op. cit., 2020, pp. 8-9.

19 Porcentaje de consejerías conseguidas con respecto al total por los diferentes PANES en las primeras elecciones a cabildos insulares. Tanto AHI como AM consiguen la mayoría abso- 


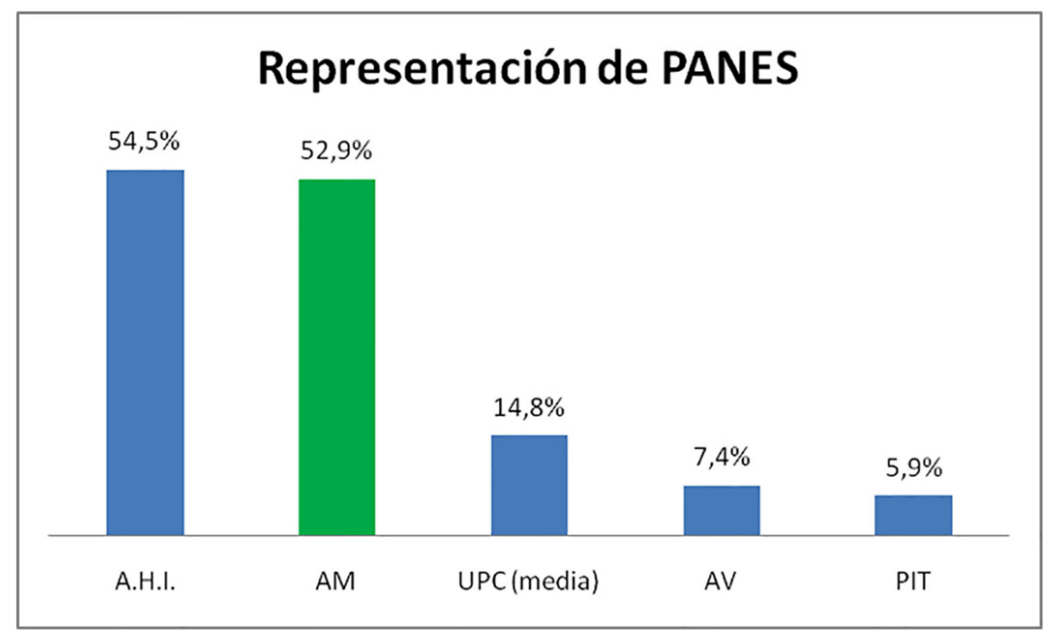

Figura 1. «Representación de PANES».

Hierro y solamente para el año $1979^{20}$. En la llamada «isla del meridiano» aparecería la AHI, consiguiendo un resultado asimilable al de AM en el ámbito insular, no así en el local, donde no presentaron candidaturas a los dos municipios existentes por ese entonces. La dinámica de los setenta en Fuerteventura era clara, la gran mayoría de instituciones se repartían entre AM y la UCD, cuando no había una lucha encarnizada entre ambas, prueba de ello es que fueron las únicas agrupaciones políticas que pudieron presentar candidatura a todos los ayuntamientos y al Cabildo ${ }^{21}$. La realidad es que fueron los dos cabildos dominados por los insularistas los únicos que sobrevivieron a la victoria aplastante de la UCD, que vencería en los otros cinco.

La victoria de Gerardo Mesa en Fuerteventura puso la primera piedra de un control casi permanente de la institución por parte de AM, ya fuera con el nombre de AM o, posteriormente, con el de Coalición Canaria. El 49,6\% de los votos cosechados en esa elección fueron el techo del partido (su suelo está en el $25 \%$ cosechado por CC en las recientes elecciones de 2019), que pudo revalidar la mayoría

luta con porcentajes prácticamente similares, mientras que la representación de la UPC (el resultado presentado es una media de los datos de las dos islas por las que consiguen representación: Tenerife y Gran Canaria), AV y el PIT, quedan a bastante distancia de los resultados cosechados por las primeras fuerzas insularistas de la Democracia en Canarias. Elaboración propia. Datos del ISTAC.

${ }^{20}$ El surgimiento de AHI es diferente al de AM, más tardío; las primeras elecciones a las que se presenta la agrupación herreña son estas del Cabildo de 1979, no concurriendo a los comicios municipales hasta las del año 1987.

${ }_{21}$ "Cuatro candidaturas para el Cabildo», en El Eco de Canarias, Las Palmas de Gran Canaria, 20/02/1979, p. 26. 
absoluta en 1983, y ser la fuerza más votada y representada, aunque no fuera con mayoría, en el resto de elecciones al Cabildo que se han producido desde entonces.

AM propicia una situación bastante extraña por lo compleja que es la transición entre un modelo político y otro. Con un nacimiento tan marcadamente asambleario, consigue adaptarse a los cambios que se producen en la dinámica democrática de la década de los ochenta, menos participativa que la anterior, generando liderazgos fuertes y llamativos que sustituyen poco a poco pero con éxito la movilización colectiva heredera de una tradición en la que los deseos de libertad se tradujeron en una sociedad bastante más movilizada. Con el descenso del flame de la Transición, AM se supo adaptar a una nueva realidad cambiante con una mayor delegación para seguir dominando la política majorera durante los ańos siguientes ${ }^{22}$.

Resulta evidente, y es una de las conclusiones que emanan de este epígrafe, que AM nace en un contexto en el que la izquierda nacional no está implantada en el territorio, pero para no resultar reiterativos (esta idea se desarrollará más adelante) y para atender también a los matices, es necesario aclarar que el éxito de AM reside también, en cierta forma, en no estancarse en el nicho de la izquierda. Si bien es cierto que el progresismo es su gran caladero de votos, su capacidad para establecer un discurso insularista le permite captar a un votante mucho más pragmático, más centrado, compitiendo de manera directa y erosionando así las bases de sus principales competidores por la hegemonía insular: la UCD y sus más directos herederos.

\section{UN PSOE QUE NO DESPEGA}

Las elecciones de la década de los setenta fueron un calvario para el prácticamente inexistente PSOE majorero, que no llegó a cosechar ningún resultado considerable. Esta «pobre» implantación de la agrupación socialista no era una cuestión de ese momento histórico, el partido nunca había cuajado en demasía en períodos anteriores. Ni siquiera durante la II República, donde solo había adquirido algo de importancia y muy tardía en la capital, Puerto Cabras (posterior Puerto del Rosario ${ }^{23}$. Esa escasa implantación tradicional, unida al largo período franquista y la rapidez de la entrada en dinámica democrática, impidió que los socialistas majoreros se mostraran electoralmente competitivos.

En los primeros comicios de 1977, el resultado fue realmente pobre. Para el Senado ni siquiera prepararon ninguna candidatura, por lo que pidieron el voto para Miguel Cabrera, candidato de AM, así como también lo harían el PCE y el

22 Luis LeÓn, Ángel Dámaso: op. cit., 2020, pp. 20-21.

${ }^{23}$ Véanse: Millares Cantero, Agustín: La Segunda República y las elecciones en la provincia de Las Palmas. Las Palmas de Gran Canaria: Mancomunidad de Cabildos. Museo Canario, 1982, pp. 38 y 50; SuÁrez Bosa, Miguel: Los trabajadores en las Canarias orientales durante el período de entreguerras: (estudio económico y social de Gran Canaria, Lanzarote y Fuerteventura). Las Palmas de Gran Canaria: Tesis doctoral leída en la Universidad de Las Palmas de Gran Canaria, 1993, p. 142. 
PSP $^{24}$. Sería la única circunscripción canaria al Senado en la que no presentarían candidato. Al Congreso la situación no fue mucho mejor. El partido de centroizquierda solo logró ser tercero con menos de mil votos y tras una UCD que barrió, multiplicando por más de seis el resultado de los socialistas (5832). Quedó tercera no solo en total, sino también en la mayoría de los municipios. Solamente pudieron auparse a la segunda posición (siempre muy por detrás de la UCD) en Pájara y Tuineje, quedando cuartos en una Betancuria que presentó un resultado muy curioso ${ }^{25}$. En segunda posición a nivel insular quedó la conservadora AP, quien sí logró superar por muy poco la barrera de los mil sufragios (1175).

¿Dónde se fueron, entonces, los más de 3000 votos que llevaron a Miguel Cabrera a ocupar su puesto de senador? Pues es cierto que una parte importante fueron al PSOE, pero otros se dividieron entre los múltiples partidos de izquierdas (PSP, PCE, etc.) y nacionalistas (PCU, PSCAN, etc.) que presentaron candidatura en la circunscripción de Las Palmas. Aun así, seguirían quedando más de un millar de votos huérfanos que no votaron iniciativas de izquierdas al Congreso. Ese voto, en gran medida, iría a la candidatura de la UCD, lo que confirma la capacidad de AM para captar voto de centro.

El considerable porcentaje de voto dividido expresado en la forma de apoyar a AM al Senado y a la UCD al Congreso deja entrever alguna cuestión que es difícil de ser explicada con las herramientas de las que disponemos. Aun así, se puede inferir que ello podría indicar la prevalencia de dos factores: por un lado, un voto marcadamente personalista (tanto a Miguel Cabrera como a Adolfo Suárez) ${ }^{26}$ que permita conjugar las dos posiciones en las dos urnas. Pero también la concepción de que ambos partidos, a pesar de pertenecer uno al centro-derecha y el otro a la izquierda, no eran percibidos como incompatibles ni lejanos al posicionamiento personal del elector.

Las elecciones de marzo de 1979 no marcarían un desarrollo muy distinto. A pesar de los dos primeros años de dinámica nacional en la que el PSOE sería el líder de la oposición al Gobierno de Suárez y que, probablemente, se produjera un mayor asentamiento de las siglas en la isla, su resultado fue igualmente malo, ya que solo pudieron crecer en cuatro votos (pasaron de 954 a 958 en la papeleta para el Congreso). Eso sí, esta vez quedando segundos en general y en todos los muni-

24 Bermúdez Suárez, Felipe y Torres Torres, Carmelo C.: op. cit., p. 22; Luis León, Ángel Dámaso: op. cit., 2020, pp. 5.

${ }^{25}$ En Betancuria, el municipio más pequeño de la isla, la UCD ganó con más de la mitad de los votos (54,3\%), solo que no le secundó AP (tercera con un 13,5\%), ni el PSOE (cuarta con un 4,4\%), sino Falange Espańola de las JONS Auténtica. El partido filofascista sacó el 21,2\% del voto válido en el municipio, un total de 77 papeletas que superaron ampliamente a la suma de votos recibidos en el resto de municipios de la isla. Fuente: datos del Ministerio del Interior.

${ }^{26}$ El apoyo electoral a la figura de Suárez funcionaría como uno de los principales aglutinadores de ciertos sectores centrados, incluso dentro de su partido, la UCD. Véase GüNTHER, Richard; Hopkin, Jonathan: "A crisis of Institutionalization: The Collapse of the UCD in Spain», en Günther, Richard, Montero Gibert José Ramón y Linz, Juan José (eds.): Political Parties: Old Concepts and New Challenges. Oxford: Oxford University Press, 2003, p. 204. 


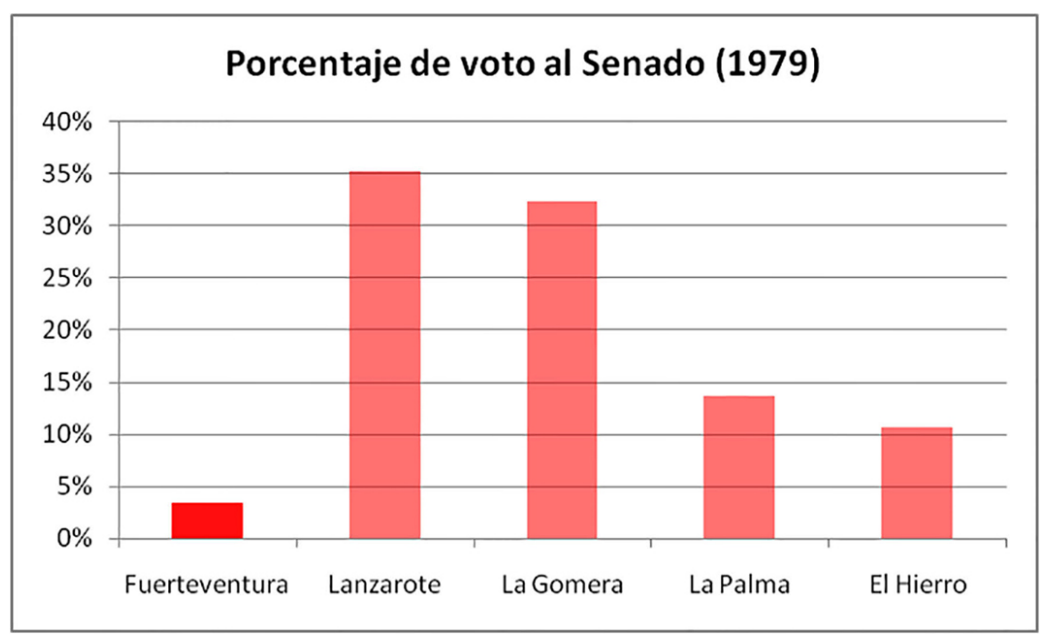

Figura 2. «Porcentaje de voto al Senado (1979)».

cipios excepto en Pájara. La escasa capacidad de movilización por parte del PSOE en Fuerteventura se puede observar en la figura $2^{27}$, donde el candidato majorero al Senado saca tres veces menos porcentaje de voto que en la siguiente isla periférica en la que menos voto reciben.

Es cierto que la candidatura del partido refleja el intento de implantarse en todo el territorio nacional, por parte de una agrupación que aspira a ocupar el Gobierno del país, pero también supone, como ya se ha podido observar anteriormente, una división del voto izquierdista que acarrea la pérdida del senador por parte de AM, en favor de la UCD, por apenas un puñado de votos.

Este mal resultado de las generales se trasladaría posteriormente a las primeras elecciones locales de la democracia, celebradas apenas un mes más tarde.

A nivel municipal, solo obtendrían representación en Tuineje, con un concejal, Melquiades de León Molina, que poco pudo hacer ante la amplia mayoría cosechada por la Asamblea de Tuineje (10 concejalías de 13). Un resultado relativamente bueno, dentro de la catastrófica tónica general, obtendrían en Antigua (6,6\% del voto válido), aunque ello no se tradujera en acta de concejal alguna. En el resto de municipios se alternarían grandes debacles con la situación de ni siquiera presentar candidatura íntegra propia, como fueron los casos de Betancuria y La Oliva ${ }^{28}$. En medio de esta debacle general, la asamblea de la militancia socialista se consolaba

27 Comparativa del porcentaje de voto cosechado por el representante del PSOE a la Cámara alta en las diferentes islas periféricas o menores. Elaboración propia. Datos del Ministerio del Interior.

28 "Cuatro candidaturas para el Cabildo", El Eco de Canarias, op. cit. 


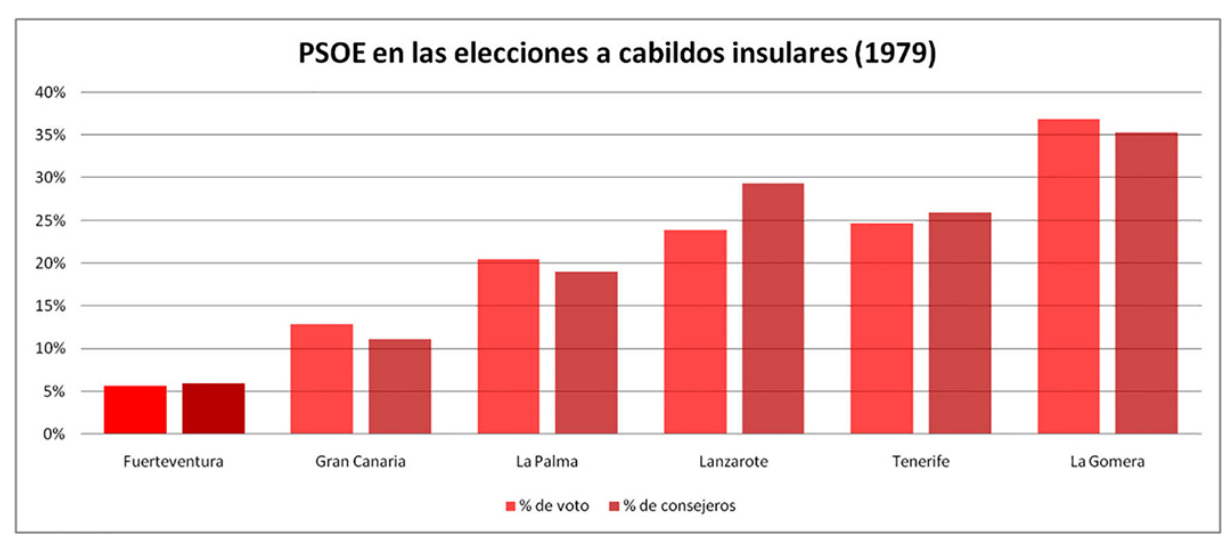

Figura 3. «PSOE en las elecciones a cabildos insulares (1979)».

afirmando que había «más concejales socialistas, aunque independientes, aportados por Asamblea Majorera a las Corporaciones» ${ }^{29}$, lo cual habla a la perfección de lo escasamente atractiva que resultaba la marca para los socialistas y socialdemócratas majoreros, que preferían acudir a las elecciones en las listas de AM.

La escasa implantación del PSOE en Fuerteventura era innegable, de los más de doscientos concejales socialistas que resultaron elegidos en toda Canarias, solo uno era majorero: un 0,005\%.

En las primeras elecciones libres para elegir el pleno del Cabildo Insular la situación fue algo mejor. En ellas, el PSOE pudo sentar a su primer consejero en el pleno, Manuel Hernández Hierro, quien pudo cosechar algo más del 5\% del voto válido y dar una de las pocas alegrías al PSOE majorero.

Dicho lo anterior y como se puede observar en el figura n. ${ }^{0} 3^{30}$, Fuerteventura presentó el resultado más pobre, tanto en voto válido como en porcentaje de consejeros, de todas las islas en las que los socialistas presentaron candidatura, cosechando casi cuatro veces menos voto válido que la siguiente isla periférica y menos de la mitad que Gran Canaria, la siguiente isla en menor apoyo a los socialistas.

Además de ello, su lista era realmente pobre en cuanto a cuadros, los cuales la mayoría aparecían en dos listas diferentes (Cabildo y Ayuntamiento). De los diez primeros candidatos de la lista socialista al Cabildo, solamente su cabeza y a posteriori consejero electo, Manuel Hernández Hierro, no redoblaba su candidatura para

29 «Relanzamiento del PSOE a nivel insular», en El Eco de Canarias, Las Palmas de Gran Canaria, 21/04/1979, p. 28.

30 Comparativa del porcentaje de votos y de escaños sobre el total, cosechados por el PSOE en las elecciones a cabildos insulares de 1979. Para El Hierro el PSOE no presentó candidatura propia. Elaboración propia. Datos del ISTAC (Instituto Canario de Estadística). 
otra institución de la isla, pero es que, además, se produce una situación muy pintoresca, ya que, de esos otros nueve candidatos que repiten, tres lo hacen no en listas municipales del PSOE, sino en listas de $\mathrm{AM}^{31}$. Ello venía a confirmar dos ideas. La primera es aquella percepción que tenían los socialistas majoreros de que había más representantes de filiación socialista en las instituciones que los resultados que había conseguido el PSOE con su marca. Y una segunda y más importante, que no es otra que la sensación de compatibilidad que tenían ambos proyectos políticos en el inicio de la democracia.

\section{LA OTRA IZQUIERDA}

Si AM pasó por estas primeras elecciones como un aluvión que no dejó lugar al PSOE, más aún pasaría sobre otros grupos de la izquierda que resistieron aún menos. En las elecciones de 1977, la suma de sufragios del resto de agrupaciones de izquierdas (entendiendo por izquierda como un segmento amplio que va desde el centro-izquierda reformista a partidos a la izquierda del eurocomunismo), unida a las de los partidos nacionalistas, apenas pudo alcanzar los 1133. Algunos votos más que los cosechados por el PSOE, pero menos que los que conseguiría AP.

Decir que sacó más que el PSOE nos puede llevar a conclusiones precipitadas. El PSOE era una sola agrupación, mientras que esos 1133 sufragios resultan de la suma de hasta siete partidos diferentes, lo que otorga una media de 161,9 votos por partido. Partiendo de ese indicador, solamente "el partido de Tierno Galván», el PSP-US (425 votos), que tuvo bastante apoyo en Puerto del Rosario, y el PSCAN (235 votos) quedaron por encima de la media. Dos partidos tan importantes como el PCC-PCE y PCU apenas cosecharon 142 y 115 sufragios, respectivamente.

En los comicios de 1979, aunque la situación empeoró para los partidos del nacionalismo y de la izquierda majorera en lo que a asignación de representantes se refiere (se perdió el senador por la isla), mejoró bastante en cuanto a votos. El voto total a partidos de izquierdas y/o nacionalistas (en un sentido amplio del término) ascendió a 1181 votos totales y una media de casi 169 votantes por agrupación. El

${ }^{31}$ Dos de los tres personajes que coinciden en dos listas de partidos diferentes son Manuel de los Reyes Monzón Hernández (5..$^{\circ}$ en la lista socialista al Cabildo y $8 .^{\circ}$ en la de AM en La Oliva) y Marcos Pérez Gutiérrez (10. ${ }^{\circ}$ en la lista socialista al Cabildo y $6 .^{\circ}$ en la de AM para el municipio de La Oliva). Ambos se presentan por el municipio de La Oliva, donde el PSOE no articula una lista para concurrir, lo que podría dar la sensación de que hay una especie de cooptación temporal en lugares donde los socialistas no se pueden presentar solos. Esta hipótesis se demuestra incorrecta o, al menos, no correcta del todo, cuando se observa que el tercero de los hombres que repiten es Manuel Sosa Roger, quien se presenta el $7 .^{\circ}$ en la lista del PSOE al Cabildo Insular, y el $5 .^{\circ}$ en la lista municipal de AM para Puerto del Rosario, municipio donde el PSOE sí que consigue presentar una lista. Como curiosidad reseñar que el único de estos «repetidores» que consigue el acta de concejal es precisamente Sosa Roger. Para contrastar, véase "Cuatro candidaturas para el Cabildo», El Eco de Canarias, op. cit. y datos del Ministerio del Interior. 
principal ascenso en estos comicios lo experimentaron la UPC, los comunistas y el efímero PDPCAN.

La UPC era un proyecto aglutinante nacionalista y de izquierdas que se había desarrollado en ese año con la agregación de varios sectores nacionalistas, marxistas y de cristianos de base ${ }^{32}$. El proyecto resultó francamente exitoso en ese ambiente de finales de década, pero rápidamente se desinfló con el paso del tiempo. En el ámbito majorero, la UPC y AM no consiguieron llegar a un acuerdo de coalición, pero sí que se produjo la petición del voto, por parte de la agrupación nacionalista, para Cabrera, para intentar evitar así que se produjera una división del voto que aupara a la derecha a ganar la contienda al Senado, como finalmente se produciría ${ }^{33}$.

De los otros dos partidos, destacar que los comunistas prácticamente doblan sus sufragios con respecto a 1977 (167 votos en esta ocasión), aupados por una mayor institucionalización del partido a nivel nacional y regional y tras una campaña bastante más activa y e implicada de los líderes regionales ${ }^{34}$. El caso del PDPCAN (o PPC) es diferente porque se sitúa en el espectro político contrario, surgiendo como una iniciativa más bien regionalista en el ámbito de la derecha ${ }^{35} \mathrm{y}$, por tanto, no sumándose al agregado total de los votos de los partidos izquierdistas y nacionalistas anteriormente mencionados.

De estos dos comicios también subyace otro aspecto crucial: no hay candidaturas de estos partidos pequeños al Senado. Esto es debido a dos cuestiones principales. Por un lado, la casi nula implantación sobre el terreno que estos tenían, quedando, en su mayoría, su ámbito de acción restringido a la isla de Gran Canaria y en algunos casos siendo pequeñas expresiones surgidas en la urbe capitalina. Por otra parte, la capacidad que tenían AM y Cabrera para hegemonizar todo el discurso y la representación de la izquierda por ese entonces hacía que estos partidos no se plantearan presentar un candidato con el que acudir a las contiendas electorales en Fuerteventura.

Esta misma situación se reproduciría un mes más tarde con los comicios locales. Al Cabildo no se presenta ninguna agrupación izquierdista que tosa a AM, solamente hay participación socialista. A nivel municipal, se produce más o menos lo mismo, ya que solamente hay una excepción: el PTIC ${ }^{36}$. Este pequeńo partido solo presenta candidatura en dos municipios concretos, Antigua y Pájara, obte-

32 Luis LeÓN, Ángel Dámaso: «Nacionalismo canario de izquierdas: la efímera Unión del Pueblo Canario", en El futuro del pasado, n. ${ }^{\circ}$ 6, 2015, pp. 293-294.

33 Ibid., p. 296.

34 "Faltan explosivos», en El Eco de Canarias, Las Palmas de Gran Canaria, 25/02/1979, p. 26.

35 El PPC es un efímero partido regionalista y de centro-derecha surgido en Las Palmas en el año 1979 y que tuvo un relativo éxito electoral en sus inicios. Con el paso del tiempo, y tras varias vicisitudes, la mayoría de sus componentes acabarían engrosando las filas del PNC. Véase: HernáNDEZ Bravo DE Laguna, Juan: «El nacionalismo y el regionalismo canarios en torno al siglo XX», en Cuadernos del Ateneo, n. ${ }^{\circ}$ 18, 2004, p. 19.

36 PTIC fue el nombre que adquirió en el archipiélago el PTE para estas elecciones. Véase Diario de Las Palmas, Las Palmas de Gran Canaria, 17/08/1989, p. 4. 
niendo pírricos resultados (dos y tres votos, respectivamente) y mostrando la incapacidad de competir localmente por parte de los partidos a la izquierda del PSOE.

Esta sombría situación podría ser explicada por una escasa implantación en todo el archipiélago, pero a poco que se contrasten los datos se ve que no es así. Echando un vistazo rápido a la dinámica de Lanzarote, la isla más cercana a Fuerteventura geográfica, cultural y sociológicamente, observamos que la realidad es diferente. Más allá del peso que ya tiene el PSOE en la isla, que es mucho mayor que en Fuerteventura, se pueden ver algunas diferencias con las agrupaciones situadas a la izquierda de los socialistas. En primer lugar, se observa cómo la UPC tiene cierto apoyo en algunas zonas de la isla, sobre todo para los comicios generales de 1979, así como la capacidad para presentar candidatos a muchas listas como la del Cabildo lanzaroteño en 1979 o la del Senado de ese mismo año, aunque no obtuvieran representación. El PCE también muestra una mayor implantación en Lanzarote que en Fuerteventura, presentando candidatos a diversos comicios como los antes mencionados para la UPC, en los cuales tampoco obtuvo representación. La potencia electoral en bruto de los comunistas lanzaroteños quizás fuera más endeble que la de los nacionalistas, pero también más concentrada, ya que en abril de 1979 entran en las instituciones cuando gana José Hernández Álvarez su acta de concejal por Arrecife ${ }^{37}$. La comparación ni siquiera se sostiene con otras islas periféricas como La Palma, donde la implantación de los comunistas será fuerte (aunque localizada), llevándole a cosechar allí los resultados más satisfactorios de los inicios de la democracia ${ }^{38}$.

En cierta medida, este análisis comparado lo que muestra es que, más que una realidad generalizada para el archipiélago o, siendo más concretos, para las llamadas «islas menores», lo que se puede observar es una relativa diferenciación interna entre cada una de ellas. El peso de los pequeños partidos nacionalistas y/o de izquierdas en Fuerteventura en los inicios de la democracia era prácticamente inexistente, no solo en términos generales, sino aún más en términos comparativos.

\section{LAS ELECCIONES DE 1982: EL CAMBIO DE CICLO}

Las últimas elecciones antes de la consolidación total del modelo autonómico supusieron un cambio crucial en la realidad política de Espańa. La implosión de una UCD que había capitaneado la Transición y el crecimiento fulgurante de un PSOE nacional (202 diputados en el Congreso, la cifra más alta de la historia de la democracia) que había atravesado con éxito los problemas internos del cambio de

37 Datos extraídos del Ministerio del Interior y de «Concejales elegidos en toda la provincia», en El Eco de Canarias, Las Palmas de Gran Canaria, 05/04/1979, p. 29.

${ }^{38}$ Martín Pérez, Nazaret: La Transición en La Palma (contribución a su legado periodistico y documental). Santa Cruz de Tenerife: Ediciones Idea, 2019, pp. 23-24. 


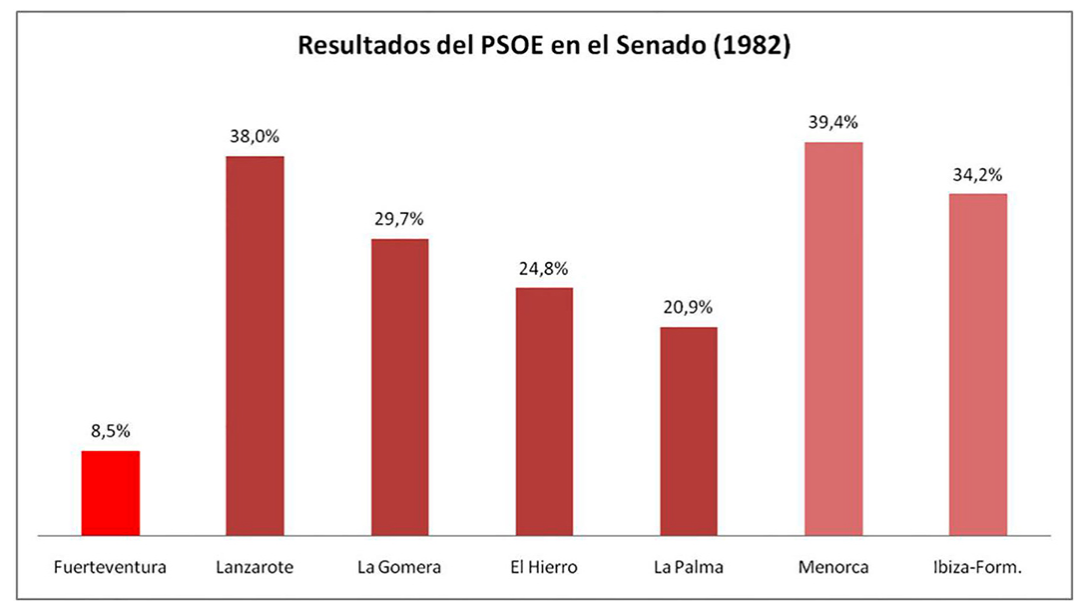

Figura 4. «Resultados del PSOE en el Senado (1982)».

decenio ${ }^{39}$ cambiaron el mapa político del país. Llegados a este punto, la pertinente pregunta sería ¿̇se produjo idéntico vuelco en el ámbito majorero?

La respuesta es un rotundo no. En el Senado, Miguel Cabrera recuperó su asiento sin mayor dificultad al crecer en casi 2000 votos. A pesar de que AM tenía experiencia captando voto centrista, no se capta prácticamente trasvase de voto desde los partidos de derechas (5184 votantes totales en 1982 por 5118 en 1979), al menos no se percibe con los datos en bruto, sino que el crecimiento de votantes totales por crecimiento poblacional se tradujo en un mayor número votos a la candidatura de Miguel Cabrera.

En cuanto al PSOE, la única agrupación de izquierda estatal que presentó candidato a la Cámara alta, experimentó un relativo crecimiento del voto. El candidato sería el consejero del Cabildo Insular, Manuel Hernández Hierro, una de las escasas figuras visibles del partido, y quien multiplicaría por tres el resultado de las últimas elecciones, pasando de poco más del 3\% a un nada desdeñable $8,5 \%$ del voto a candidaturas.

A pesar de este crecimiento del voto a la agrupación socialista majorera, el peso del partido en la isla seguía siendo muy escaso en términos comparativos. Como se puede observar en la figura n. ${ }^{\circ} 4^{40}$, el resultado cosechado en Fuerteventura seguía suponiendo el menor porcentaje de voto al PSOE, en papeletas sepia,

39 Tusell, Javier: op. cit., pp. 327-328.

${ }^{40}$ Comparativa del porcentaje de voto válido cosechado por el PSOE en las islas no capitalinas de los dos archipiélagos españoles, para elecciones al Senado de 1982. Elaboración propia. Datos del Ministerio del Interior y del ISTAC. 
de todas las islas no capitalinas, no solo de Canarias, sino de los dos archipiélagos espańoles, y la diferencia con el resto continuaba siendo considerable.

Además del escaso peso relativo del PSOE en la isla, también se produjo otra dificultad, el crecimiento del partido a nivel nacional tiró hacia arriba del voto en la isla, pero no en igual proporción. Este hecho no solo se produjo en los votos al Senado, sino que también pasaría al Congreso.

Fuerteventura sería uno de los escasos lugares del país donde no ganarían los socialistas. La victoria sería para uno de los herederos del centrismo: el CDS. Se dice uno porque su victoria tuvo incluso más mérito al competir en sus espacios electorales, el centro y la derecha, con varios competidores, entre ellos la propia marca UCD, o lo que quedaba de la misma. Esta victoria del CDS sobre otros grupos de derecha y centro-derecha apuntala un poco más la hipótesis de que el gran aglutinador del voto majorero en los primeros años era la figura de Adolfo Suárez.

En estas condiciones, se puede decir que el crecimiento del PSOE fue importante, ya que incluso ganaría en municipios como Pájara, Antigua o Tuineje, pero bastante limitado, sobre todo en términos comparativos. Si se ponen sobre la mesa, por ejemplo, los resultados majoreros y los lanzaroteños, la diferencia es abismal. En la «isla de los volcanes», el PSOE no solo ganaría en la gran mayoría de los municipios, sino que además, en términos generales, doblaría en voto total al segundo partido más votado, la UCD.

Más allá del crecimiento experimentado por el PSOE en Fuerteventura, también se produjo una subida general y notable de la izquierda organizada en sus formas más habituales. La UPC también creció bastante (en Pájara serían segunda fuerza en votantes solamente por detrás del PSOE), llegando a rozar el millar y medio de electores. Una cifra impensable en años anteriores. Esta subida pudo estar determinada tanto por su mayor implantación territorial como, sobre todo, por un efecto arrastre por parte de AM.

En resumidas cuentas y a pesar de los cambios en las siglas y las suavizaciones de las tendencias, lo que se puede observar en las elecciones generales de 1982 es la misma dinámica que en las anteriores, las izquierdas vencen en las elecciones al Senado, mientras que las derechas ganan al Congreso. Esta situación confirma la cantidad de split ticket que se produce en el contexto majorero durante los inicios de la democracia, sobre todo cuando se producen elecciones generales, no tanto en las locales.

En realidad, lo que se puede observar son los primeros pasos de un modelo político que se desarrollará en los años siguientes y es la amplia vocación insularista del electorado majorero, que tendrá a un insularista gobernando ininterrumpidamente el Cabildo desde 1979 hasta el año 2019, cuando a pesar de ganar las elecciones $\mathrm{CC}$ no consigue articular mayoría ${ }^{41}$. Esto ya venía de antes porque no solo AM tenía esta vocación insularista, también existía en la esencia de la UCD, ger-

41 «Presentada la moción de censura contra Lola García en el Cabildo de Fuerteventura», en Diario de Fuerteventura, Puerto del Rosario, 24/06/2019, disponible en https://www.diariode- 
men de lo que luego serían los $\mathrm{IF}^{42}$, quienes consideraban que la comunidad autónoma debía construirse desde los cabildos hacia el centro, primando a la isla, y no a la inversa. Llama la atención que sean precisamente estas dos agrupaciones, con visiones desde la izquierda y desde el centro-derecha del insularismo, las que dominen totalmente la dinámica electoral de la isla en los inicios del período democrático.

\section{CONCLUSIONES}

A modo de conclusiones, se pueden extraer una serie de ideas que destacan tras el análisis de los datos presentados y que en última instancia deben responder a la cuestión principal planteada en el inicio del artículo y que se retoma a continuación: ¡es Fuerteventura una realidad diferenciada? Pues con los datos y el análisis realizado en la mano se debe decir que sí.

La respuesta a cuál sería el elemento diferenciador que separaría a la dinámica partidista majorera del resto es, sin duda alguna, Asamblea Majorera. El surgimiento de AM y, sobre todo, el contexto temprano del surgimiento de AM es lo que determina esa diferenciación majorera, así como las tres conclusiones principales que emanan de este proceso investigador.

Antes de desarrollar las mismas, hay que hacer un necesario inciso de carácter institucional. Todo lo que se produce en esta etapa está propiciado por la dinámica institucional creada en ese contexto y que es heredera del período previo. Es la pervivencia de los cabildos insulares y la implantación de un Senado que reconoce a la isla como entidad territorial canaria de base lo que propicia el surgimiento de AM. Aun así, destaca no solo la rapidez con la que el partido se monta y aglutina votantes, sino sobre todo su articulación desde la izquierda del espectro político.

Retomando las conclusiones, la primera de las que emanan es que Fuerteventura, en los inicios de la democracia, es un paraíso del split ticket y ello está provocado directamente por AM. La incapacidad de AM de ser competitivo en unas elecciones al Congreso, por cuestiones obvias de peso poblacional de la isla (y de no aparición de alternativas similares en la provincia con las que coaligarse), hacía que no se presentaran a estos comicios, reclamando el voto para otras agrupaciones, que no satisfacían en gran medida a su electorado, y propiciando así resultados diferenciados para las dos cámaras. Esta situación, unida a su capacidad para ser hipercompetitivos en las elecciones al Senado, hacía que muchos electores de la agrupación tuvieran que decidir con qué acompañaban a su voto insularista en la otra papeleta.

La segunda es la lentitud con la que el PSOE se convierte en alternativa competitiva para el poder. Es cierto que el condicionante principal para que el PSOE

fuerteventura.com/noticia/presentada-la-moci $\% \mathrm{C} 3 \% \mathrm{~B} 3 \mathrm{n}$-de-censura-contra-lola-garc\%C3\%ADaen-el-cabildo-de-fuerteventura (consultado el 28-07-2019).

${ }^{42}$ García Rojas, José Adrián: Un caso de competencia electoral subestatal: la competencia electoral en Canarias. Madrid: Departamento de Ciencia Política y de la Administración de la Universidad Complutense de Madrid, 2002, p. 142; Luis León, Ángel Dámaso: op. cit., 2020, p. 15. 
no sea un partido competitivo en los primeros comicios celebrados es su escasa tradición histórica en la isla, pero será la aparición de AM, que se constituye como el principal canalizador del voto del centro-izquierda, lo que termine por ejercer de tapón a su crecimiento natural. Además de ello, y hasta más importante, es que no solo no permite una articulación temprana en la incipiente década de los setenta, sino que actúa como freno para la oleada expansiva que la agrupación del puño y la rosa experimentará a principios de los ochenta, aupada por el fenómeno nacional, siendo uno de los territorios de todo el país donde los socialistas se desarrollarán en menor cantidad durante la primera década de la democracia.

Si eso genera AM con el principal partido de la izquierda, qué no le producirá al resto de agrupaciones del espectro. Los partidos a la izquierda del PSOE se mostraron aún menos fuertes que este porque también se produce una hegemonía del discurso por parte de AM, que lo representaría, en muchas ocasiones, con más éxito y desde las instituciones. Al mal socialista de la poca implantación histórica y del aplastamiento por parte de AM en la contienda electoral habría que ańadirle, para la casuística de este tipo de agrupaciones, otra serie de condicionantes que le son propios: tamańo, poca implantación en entornos no urbanos, división en múltiples grupos, etc., situación que sin ninguna duda hace que la mayoría queden vistos por el pueblo majorero como innecesarios, irrelevantes o, en el peor de los casos, como foráneos. 


\section{ANEXO I. ÍNDICE DE SIGLAS}

AHI Agrupación Herreña Independiente

AM Asamblea Majorera

AP Alianza Popular

AV Asamblea de Vecinos

CC Coalición Canaria

CD Coalición Democrática

CDS Centro Democrático y Social

IF Independientes de Fuerteventura

PCC-PCE Partido Comunista de Canarias-Partido Comunista de España

PCU Pueblo Canario Unido

PIT Partido de Independientes de Teguise

PNC Partido Nacionalista de Canarias

PPC (o PDPCAN) Partido del País Canario

PSC-PSOE Partido Socialista de Canarias-Partido Socialista Obrero Español

PSCAN Partido Socialista de Canario

PSP-US Partido Socialista Popular-Unidad Socialista

PTE-PTIC Partido de los Trabajadores de España-Partido de los Trabajadores de las Islas Canarias

UCD Unión de Centro Democrático

UPC Unión del Pueblo Canario 